\title{
COMPOSIÇÃO DA FLORA ARBÓREA DE UM TRECHO DE FLORESTA ESTACIONAL NO JARDIM BOTÂNICO DA UNIVERSIDADE FEDERAL DE VIÇOSA (FACE SUDOESTE), VIÇOSA, MINAS GERAIS ${ }^{1}$
}

\author{
Waldomiro de Paula Lopes ${ }^{2}$, Alessandro de Paula ${ }^{2}$, Anderson Cassio Sevilha ${ }^{3}$ e Alexandre Francisco da Silva ${ }^{4}$
}

\begin{abstract}
RESUMO - Estudou-se um fragmento de Floresta Estacional Semidecidual Submontana, localizado no município

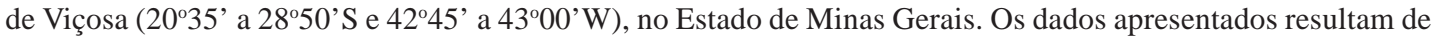
uma amostragem fitossociológica realizada em uma área de 1 hectare quadrado. Foram relacionados todos os indivíduos arbóreos que apresentavam circunferência de tronco à altura do peito maior ou igual a $15 \mathrm{~cm}$, o que resultou em 121 espécies, compreendendo 89 gêneros, distribuídos em 39 famílias. Considerando os gêneros, as famílias mais bem representadas foram Leguminosae (16), Rubiaceae (6) e Euphorbiaceae e Myrtaceae, com cinco cada uma. As famílias mais ricas em espécies foram Leguminosae (20), Lauraceae (10), Rubiaceae (9) e Flacourtiaceae (8). Dentre os gêneros encontrados, destacaram-se Ocotea e Casearia, com sete e cinco espécies, respectivamente. Entre os vários fragmentos analisados na região, o trecho de floresta estudado apresentou-se como o mais rico em espécies, considerando o mesmo critério de amostragem.
\end{abstract}

Palavras-chave: Floresta Estacional Semidecidual Submontana, composição florística, flora arbórea, Zona da Mata mineira.

\section{COMPOSITION OF THE ARBOREAL FLORA OF A STRETCH OF THE SEMIDECIDUOUS FOREST OF THE BOTANICAL GARDEN AT THE UNIVERSIDADE FEDERAL DE VIÇOSA (SOUTHWEST SIDE), VIÇOSA, MINAS GERAIS, BRAZIL}

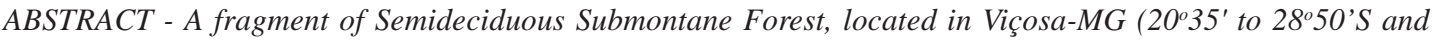
$42^{\circ} 45^{\prime}$ to $43^{\circ} 00^{\prime} \mathrm{W}$ ), was studied. The data presented are the result of a phytosociological analysis carried out in an area of an hectare square. All trees wich a minimum trunk circunference of $0.15 \mathrm{~m}$ at $1.30 \mathrm{~m}$ from the soil level were sampled, comprising 121 species of 89 genera, distributed over 39 families. In regards to genera, the families most represented were Leguminosae (16), Rubiaceae, (six) and Euphorbiaceae and Myrtaceae, each with five genera. In regards to number of species, the most represented were Leguminosae (20), Lauraceae (ten), Rubiaceae (nine) and Flacourtiaceae (eight). The genera Ocotea and Casearia were the most prominent, showing seven and five species, respectively. Among the several stretches studied in the same region, the one presently studied had the largest number of especies, when the same sample criterion was adopted.
\end{abstract}

Key words: Semideciduous submontane forest, floristic composition, arboreal flora, Zona da Mata, Minas Gerais.

1 Recebido para publicação em 20.11.2000.

Aceito para publicação em 20.6.2002.

Auxílio financeiro do CNPq

2 Engenheiro Florestal - M.S. Botânica. ${ }^{3}$ Biólogo - M.S. Botânica. ${ }^{4}$ Departamento de Biologia Vegetal da Universidade Federal de Viçosa - UFV, 36571-000 Viçosa-MG, <afsilva@ufv.br>. 


\section{INTRODUÇÃO}

Em suas condições originais, a formação vegetal que sugeriu o nome da Zona da Mata de Minas Gerais formava um todo contínuo com a floresta do médio Paraíba, ao sul, e a do vale do rio Doce, ao norte; a oeste limitavamna os campos naturais do centro e sul de Minas (Valverde, 1958). A cobertura florestal original manteve-se pouco alterada até o início do século XIX. A partir dessa época ocorreu a efetiva ocupação humana da região, por meio de um processo predatório, com o corte raso da floresta, visando destinar áreas para a cafeicultura, pecuária ou simplesmente a comercialização da madeira. Apenas as áreas de difícil acesso permaneceram com menor nível de intervenção antrópica.

Esses fatores foram decisivos para gerar o padrão de distribuição das florestas encontradas atualmente na Zona da Mata de Minas Gerais, em pequenos fragmentos secundários. Portanto, tem-se uma situação preocupante em função das implicações ecológicas relacionadas a essa fragmentação, como comentou Wilcove (1986), que considerou previsível a extinção de espécies em uma dada comunidade, devido à perda em biodiversidade, ao tamanho reduzido das áreas, ao efeito dos habitats ao redor do fragmento e ao efeito de borda.

Oliveira-Filho \& Machado (1993) comentaram que, apesar da crescente consciência acerca da importância desses recursos florestais e da urgência de conservá-los, pouco se sabe sobre a composição florística dos remanescentes existentes no centro-sul e leste de Minas Gerais, que ainda representam parte da vegetação original.

Os objetivos do presente trabalho foram conhecer a composição florística de um fragmento de floresta secundária na Zona da Mata de Minas Gerais, que se encontra sob processo de regeneração natural há cerca de 70 anos, e fornecer dados sobre grupos ecológicos das espécies.

\section{MATERIAL E MÉTODOS}

O município de Viçosa localiza-se entre as coordenadas de $20^{\circ} 35^{\prime}$ a $28^{\circ} 50^{\prime} \mathrm{S}$ e $42^{\circ} 45^{\prime}$ a $43^{\circ} 00^{\prime} \mathrm{W}$, no sudeste do Estado de Minas Gerais, em região caracteristicamente montanhosa. A região faz parte das Terras Altas Brasileiras, em uma divisão geopolítica denominada de Zona da Mata. Os solos da região apresentam-se com baixa fertilidade, sendo classificados como Latossolo Vermelho-Amarelo e Latossolo Amarelo, predominantemente (Valverde, 1958).

R. Árvore, Viçosa-M G, v.26, n.3, p.339-347, 2002
O clima, segundo a classificação de Köppen, é do tipo Cwa, mesotérmico úmido com verões chuvosos e invernos secos (Vianello \& Alves, 1991), com défice hídrico no período de maio a setembro e um excedente entre dezembro e março (Golfari, 1975). As médias anuais de precipitação pluvial, umidade relativa do ar e temperatura do ar são, respectivamente, $1.340 \mathrm{~mm}, 80 \%$ e $19{ }^{\circ} \mathrm{C}$ (CASTRO et al., 1973).

A área de estudo, com face de exposição nordeste, situa-se no Jardim Botânico da Universidade Federal de Viçosa (UFV), no interior do campus da Universidade, a uma altitude em torno dos $700 \mathrm{~m}$ e com área aproximada de 75 ha. A listagem florística foi elaborada a partir de uma amostragem fitossociológica de 1 hectare quadrado, subdividido em 100 parcelas de $10 \times 10 \mathrm{~m}$, das quais 55 foram alocadas em uma encosta (inclinação igual ou maior que $10 \%$ ) e 45 no platô (inclinação menor que 10\%). O critério de inclusão adotado foi de indivíduos arbóreos que apresentavam circunferência de tronco a 1,30 $\mathrm{m}$ do solo (CAP) igual ou maior que $15 \mathrm{~cm}$. O local de estudo é permanentemente protegido, estando sob processo de regeneração natural desde 1926, quando foi abandonado um cafezal implantado após corte raso da floresta.

Segundo Veloso et al. (1991), o ambiente florestal avaliado pode ser classificado como Floresta Estacional Semidecidual. Com relação ao tipo de formação, optouse por considerar a alteração adotada por Oliveira-Filho et al. (1994), que propuseram o limite altitudinal de $750 \mathrm{~m}$ entre as formações submontana e montana, nas latitudes entre 16 e $24^{\circ} \mathrm{S}$.

Para apresentação das famílias e respectivas espécies, tomou-se como referência o sistema de classificação de Cronquist (1981), exceto para as famílias Caesalpiniaceae, Fabaceae e Mimosaceae, que foram mantidas como Leguminosae e respectivas subfamílias. A sinonímia e a grafia dos taxa foram realizadas mediante consulta ao índice de espécies do Royal Botanic Gardens - KEW (1997), ou literatura mais recente. Quanto aos autores, utilizou-se a padronização recomendada por Brumit \& Powell (1992).

As espécies encontradas no presente levantamento foram atribuídas ao grupo ecológico de acordo com Gandolfi (1991), que as distinguiu como pioneiras, secundárias iniciais e secundárias tardias. Dados referentes às espécies foram também tomados dos trabalhos de Oliveira-Filho et al. (1994, 1997) e de Paula (1999). As discordâncias existentes nessa classificação se devem a observações de campo dos autores do presente trabalho. 
Todo material coletado foi determinado no setor de Taxonomia do Departamento de Biologia Vegetal da Universidade Federal de Viçosa, em alguns casos sendo enviado a especialistas. As exsicatas foram incorporadas ao acervo do Herbário VIC.

\section{RESULTADOS E DISCUSSÃO}

O fragmento florestal estudado possui o estrato herbáceo-arbustivo bastante denso. Além de representantes jovens das espécies dos estratos arbóreos, encontram-se com freqüência espécies de Ruellia e Justicia (Acanthaceae), Piper e Ottonia (Piperaceae), Psychotria e Palicourea (Rubiaceae) e Polypodium (Polypodiaceae), Blechnum (Blechnaceae) e Adiantum (Pteridaceae), que se desenvolvem nos locais mais úmidos e sombreados. Por sua vez, Capsicum e Cyphomandra (Solanaceae) e Triumphetta (Tiliaceae) são comuns nas áreas mais iluminadas e secas. Também são observadas diversas espécies de lianas, com ocorrência considerável, principalmente em trechos correspondentes a clareiras mais recentes.

Espécies exóticas como Citrus sp., Coffea arabica L. e Eryobotria japonica Lindl. estão presentes na área de estudo, possivelmente introduzidas por dispersores naturais ou, acidentalmente, pela ação antrópica. Em relação a $C$. arabica, sua presença é considerada fator negativo, tanto para os representantes de espécies típicas dos estratos herbáceo e arbustivo, como para a regeneração das espécies arbóreas que ocorrem nos demais estratos da floresta. Situação semelhante foi encontrada por Martins (1991), que observou na Mata Capetinga (Parque Estadual de Vassununga-SP) a ocorrência de pequenas áreas em que as plantas jovens dessa espécie formavam magotes quase puros. $\mathrm{O}$ autor referiu-se à ação de inibidores químicos da germinação, disseminados pelas plantas de $C$. arabica, podendo dificultar ou, pelo menos, retardar a regeneração de populações de outras espécies na floresta, sensíveis àquele determinado inibidor.

A identificação dos indivíduos incluídos na amostragem fitossociológica resultou em 121 espécies, pertencentes a 89 gêneros reconhecidos, 38 famílias de Magnoliopsida e uma de Liliopsida (Arecaceae, representada por Attalea dubia). As famílias com o maior número de gêneros foram Leguminosae (16), Rubiaceae com seis e Euphorbiaceae e Myrtaceae com cinco cada uma (Quadro 1). As famílias Flacourtiaceae, Lauraceae e Moraceae estiveram representadas por quatro gêneros cada uma. As famílias mais ricas em espécies foram Leguminosae (20), Lauraceae (10), Rubiaceae (9), Flacourtiaceae (8), Myrtaceae (7) e Euphorbiaceae (6).

Do número total de famílias encontradas, 16 foram relacionadas com apenas uma espécie, correspondendo a 39\% do total (Apocynaceae, Aquifoliaceae, Arecaceae, Asteraceae, Caricaceae, Cecropiaceae, Ebenaceae, Erythroxylaceae, Guttiferae, Icacinaceae, Lacistemaceae, Olacaceae, Rhamnaceae, Rosaceae, Solanaceae e Tiliaceae).

No mesmo município e com o mesmo critério de inclusão utilizado no presente trabalho, SILVA et al. (2000) encontraram para 1 ha, no mesmo fragmento do presente levantamento, porém com face de exposição solar oeste, 91 espécies arbóreas, sendo Leguminosae a família mais rica (16 espécies), seguida de Lauraceae, Myrtaceae e Rubiaceae (6 cada uma) e Flacourtiaceae (5). Senra (2000), também para 1 ha, determinou 107 espécies, sendo mais ricas as famílias Leguminosae com 17 espécies, Annonaceae (9), Euphorbiaceae (8), Lauraceae (7), Flacourtiaceae e Sapindaceae (6 espécies cada uma) e Myrtaceae com (5). Soares Júnior (2000), em 200 pontos-quadrantes, encontrou 83 espécies, sendo mais ricas as famílias Leguminosae (15 espécies), Lauraceae (10) e Myrtaceae (7). Meira-Neto \& Martins (2000), com critério de inclusão de CAP igual ou superior a $10 \mathrm{~cm}$, encontraram 154 espécies em 1 ha de amostragem, tendo Leguminosae apresentado o maior número de espécies (26), seguida de Rubiaceae (16), Lauraceae (10), Euphorbiaceae e Myrtaceae (9 cada uma), Annonaceae, Flacourtiaceae e Melastomataceae (6 espécies cada), além de Rutaceae com cinco espécies; o destaque de Rubiaceae e Melastomataceae tem o critério de inclusão adotado como fator decisivo, uma vez que essas duas famílias ocorreram com muitas espécies de pequeno porte, características da submata.

Ainda na Zona da Mata mineira, em uma área de 0,35 ha no campus da Universidade Federal de Juiz de Fora, Almeida \& Souza (1997) relacionaram 78 espécies arbóreas com CAP mínimo de $15,7 \mathrm{~cm}$, destacando-se as famílias Leguminosae com 11 espécies, Euphorbiaceae (7), Myrtaceae (6) e Lauraceae (5). No município de Ponte Nova, aplicando 100 pontos-quadrantes e com critério de inclusão de CAP maior ou igual a $15 \mathrm{~cm}$, Meira-Neto et al. (1997) encontraram 89 espécies de porte arbóreo, sendo Leguminosae a família mais rica, com 20 espécies, seguida de Moraceae (6) e Rubiaceae (5). 
Quadro 1 - Espécies amostradas no Jardim Botânico da Universidade Federal de Viçosa (face sudoeste) - Viçosa, Minas Gerais, acompanhadas dos respectivos grupos ecológicos (GE). PI = pioneira, $\mathrm{SI}=$ secundária inicial, $\mathrm{ST}$ = secundária tardia, $\mathrm{IND}=$ indiferente e $\mathrm{SC}=$ sem classificação

Table 1 - Species sampled at the Botanical Garden of the Universidade Federal de Vicosa (southwest side) - Vicosa, Minas Gerais, followed by their respective ecological groups (GE). $P I=$ pioneer, $S I=$ initial secondary, $S T=$ late secondary, IND = indifferent and $S C=$ non classified

\begin{tabular}{|c|c|c|}
\hline Família & Espécie & GE \\
\hline ANACARDIACEAE & $\begin{array}{l}\text { Astronium fraxinifolium Schott } \\
\text { Tapirira guianensis Aubl. }\end{array}$ & $\begin{array}{l}\text { ST } \\
\text { SI }\end{array}$ \\
\hline ANNONACEAE & $\begin{array}{l}\text { Guatteria australis A.St.-Hil. } \\
\text { Guatteria nigrescens Mart. } \\
\text { Guatteria villosissima A.St.-Hil. } \\
\text { Rollinia laurifolia } \text { Schltdl. } \\
\text { Rollinia silvatica Mart. }\end{array}$ & $\begin{array}{l}\text { ST } \\
\text { ST } \\
\text { SI } \\
\text { SI } \\
\text { SI }\end{array}$ \\
\hline APOCYNACEAE & Tabernaemontana laeta Mart. & $\mathrm{PI}$ \\
\hline AQUIFOLIACEAE & Ilex cerasifolia Reissek & ST \\
\hline ARECACEAE & Attalea dubia Burret & PI \\
\hline ASTERACEAE & Vernonia diffusa Less. & PI \\
\hline BIGNONIACEAE & $\begin{array}{l}\text { Jacaranda macrantha Cham. } \\
\text { Sparattosperma leucanthum K.Schum. } \\
\text { Tabebuia chrysotricha (Mart. ex DC.) Standl. }\end{array}$ & $\begin{array}{l}\text { PI } \\
\text { PI } \\
\text { PI }\end{array}$ \\
\hline BOMBACACEAE & $\begin{array}{l}\text { Ceiba speciosa (A.St.-Hil.) Gibbs \& Semir } \\
\text { Eriotheca candolleana (K.Schum.) A.Robyns } \\
\text { Pseudobombax grandiflorum (Cav.) A.Robyns }\end{array}$ & $\begin{array}{l}\text { SI } \\
\text { SI } \\
\text { SI }\end{array}$ \\
\hline BURSERACEAE & $\begin{array}{l}\text { Protium warmingianum March. } \\
\text { Protium widgrenii Engl. }\end{array}$ & $\begin{array}{l}\text { ST } \\
\text { SI }\end{array}$ \\
\hline CARICACEAE & Jacaratia spinosa A. DC. & SI \\
\hline CECROPIACEAE & Cecropia glaziovii Snethl. & $\mathrm{PI}$ \\
\hline CELASTRACEAE & $\begin{array}{l}\text { Maytenus aquifolium Mart. } \\
\text { Maytenus robusta } \text { Reissek }\end{array}$ & ST \\
\hline CHRYSOBALANACEAE & $\begin{array}{l}\text { Hirtella hebeclada Moric. } \\
\text { Licania spicata Hook.f. }\end{array}$ & $\begin{array}{l}\text { SI } \\
\text { SI }\end{array}$ \\
\hline EBENACEAE & Diospyros sp. & SC \\
\hline ERYTHROXYLACEAE & Erythroxylum pelleterianum A.St.-Hil. & SI \\
\hline EUPHORBIACEAE & $\begin{array}{l}\text { Alchornea glandulosa Poepp. \& Endl. } \\
\text { Alchornea triplinervia Müll.Arg. } \\
\text { Aparisthmium cordatum Baill. } \\
\text { Croton urucurana Baill. } \\
\text { Mabea fistulifera Mart. } \\
\text { Sapium glandulosum } \text { (L.) Morong }\end{array}$ & $\begin{array}{l}\text { SI } \\
\text { PI } \\
\text { SI } \\
\text { PI } \\
\text { PI } \\
\text { PI }\end{array}$ \\
\hline FLACOURTIACEAE & $\begin{array}{l}\text { Carpotroche brasiliensis Endl. } \\
\text { Casearia arborea } \text { Urb. } \\
\text { Casearia decandra Jacq. } \\
\text { Casearia gossypiosperma Briq. } \\
\text { Casearia sylvestris } \text { Sw. } \\
\text { Casearia ulmifolia Cambess. } \\
\text { Prockia crucis } \mathrm{L} \text {. } \\
\text { Xylosma prockia } \text { (Turcz.) Turcz. }\end{array}$ & $\begin{array}{l}\text { ST } \\
\text { SI } \\
\text { SI } \\
\text { SI } \\
\text { SI } \\
\text { SI } \\
\text { SI } \\
\text { SI }\end{array}$ \\
\hline
\end{tabular}


Quadro 1, Cont.

Table 1, Cont.

\begin{tabular}{|c|c|c|}
\hline Família & Espécie & GE \\
\hline GUTTIFERAE & Mahurea exstipulata Benth. & $\mathrm{SC}$ \\
\hline ICACINACEAE & Citronella megaphylla (Miers) Howard & $\mathrm{SC}$ \\
\hline LACISTEMACEAE & Lacistema pubescens Mart. & SI \\
\hline LAURACEAE & $\begin{array}{l}\text { Aniba firmula Mez } \\
\text { Endlicheria paniculata (Spreng.) J.F.Macbr. } \\
\text { Nectandra lanceolata Nees \& Mart. ex Nees } \\
\text { Nectandra oppositifolia } \mathrm{Nees} \\
\text { Ocotea } \text { corymbosa (Meissn.) Mez } \\
\text { Ocotea dispersa } \mathrm{Mez} \\
\text { Ocotea minarum Mart. ex Nees } \\
\text { Ocotea odorifera (Vell.) Rohwer } \\
\text { Ocotea pubescens } \mathrm{Mez} \\
\text { Ocotea spixiana } \mathrm{Mez}\end{array}$ & $\begin{array}{l}\text { ST } \\
\text { ST } \\
\text { ST } \\
\text { SI } \\
\text { SI } \\
\text { SI } \\
\text { ST } \\
\text { ST } \\
\text { ST } \\
\text { ST }\end{array}$ \\
\hline LECYTHIDACEAE & $\begin{array}{l}\text { Cariniana estrellensis Kuntze } \\
\text { Cariniana legalis Kuntze }\end{array}$ & $\begin{array}{l}\text { ST } \\
\text { ST }\end{array}$ \\
\hline LEGUMINOSAE CAESALPINIOIDEAE & $\begin{array}{l}\text { Apuleia leiocarpa J.F.Macbr. } \\
\text { Cassia ferruginea } \text { Schrad. ex DC. } \\
\text { Copaifera langsdorffii Desf. } \\
\text { Peltophorum dubium Taub. }\end{array}$ & $\begin{array}{l}\text { SI } \\
\text { SI } \\
\text { SI } \\
\text { SI }\end{array}$ \\
\hline LEGUMINOSAE PAPILIONOIDEAE & $\begin{array}{l}\text { Andira fraxinifolia Benth. } \\
\text { Dalbergia nigra Allem. ex Benth. } \\
\text { Lonchocarpus muehlbergianus Hassl. } \\
\text { Machaerium brasiliense Vogel } \\
\text { Machaerium floridum Ducke } \\
\text { Machaerium stipitatum (DC.) Vogel } \\
\text { Ormosia arborea Harms }\end{array}$ & $\begin{array}{l}\text { SI } \\
\text { SI } \\
\text { ST } \\
\text { SI } \\
\text { SI } \\
\text { SI } \\
\text { SC }\end{array}$ \\
\hline LEGUMINOSAE MIMOSOIDEAE & $\begin{array}{l}\text { Acacia glomerosa Benth. } \\
\text { Albizia polycephala (Benth.) Killip } \\
\text { Anadenanthera colubrina (Vell.) Brenan } \\
\text { Inga alba Willd. } \\
\text { Inga vera Willd. } \\
\text { Pseudopiptadenia contorta } \text { (DC.) G.P.Lewis \& M.P.Lima } \\
\text { Piptadenia gonoacantha J.F.Macbr. } \\
\text { Piptadenia paniculata Benth. } \\
\text { Plathymenia foliolosa Benth. }\end{array}$ & $\begin{array}{l}\text { SI } \\
\text { SI } \\
\text { SI } \\
\text { SI } \\
\text { SI } \\
\text { SI } \\
\text { PI } \\
\text { PI } \\
\text { SI }\end{array}$ \\
\hline MELASTOMATACEAE & $\begin{array}{l}\text { Miconia cinnamomifolia Naudin } \\
\text { Miconia pauciflora Triana }\end{array}$ & $\begin{array}{l}\text { PI } \\
\text { SC }\end{array}$ \\
\hline MELIACEAE & $\begin{array}{l}\text { Cabralea canjerana (Vell.) Mart. } \\
\text { Cedrela fissilis Vell. } \\
\text { Trichilia catigua A.Juss. } \\
\text { Trichilia lepidota } \text { Sw. } \\
\text { Trichilia pallida } \text { Sw. }\end{array}$ & $\begin{array}{l}\text { SI } \\
\text { SI } \\
\text { ST } \\
\text { ST } \\
\text { SI }\end{array}$ \\
\hline MONIMIACEAE & $\begin{array}{l}\text { Mollinedia floribunda Tul. } \\
\text { Siparuna guianensis Aubl. }\end{array}$ & $\begin{array}{l}\text { ST } \\
\text { IND }\end{array}$ \\
\hline
\end{tabular}


Quadro 1, Cont.

Table 1, Cont.

\begin{tabular}{|c|c|c|}
\hline Família & Espécie & GE \\
\hline MORACEAE & $\begin{array}{l}\text { Brosimum glaziovii Taub. } \\
\text { Ficus enormis (Mart. ex Miq.) Miq. } \\
\text { Maclura tinctoria D.Don ex Steud. } \\
\text { Sorocea bonplandii (Baill.) Burger, Lanj. \& Boer }\end{array}$ & $\begin{array}{l}\text { SI } \\
\text { ST } \\
\text { SI } \\
\text { SI }\end{array}$ \\
\hline MYRTACEAE & $\begin{array}{l}\text { Campomanesia xanthocarpa Berg } \\
\text { Eugenia brasiliensis Lam. } \\
\text { Eugenia pyriformis } \text { Cambess. } \\
\text { Eugenia uniflora } \text { L. } \\
\text { Myrcia fallax DC. } \\
\text { Myrciaria axillaris } \text { Berg } \\
\text { Plinia glomerata } \text { (Berg) Amshoff }\end{array}$ & $\begin{array}{l}\text { ST } \\
\text { ST } \\
\text { SC } \\
\text { ST } \\
\text { SI } \\
\text { SI } \\
\text { SI }\end{array}$ \\
\hline NYCTAGINACEAE & $\begin{array}{l}\text { Guapira opposita (Vell.) Reitz } \\
\text { Guapira tomentosa (Casar.) Lundell }\end{array}$ & $\begin{array}{l}\text { SI } \\
\text { SI }\end{array}$ \\
\hline OLACACEAE & Heisteria silvianii Schwacke & $\mathrm{ST}$ \\
\hline RHAMNACEAE & Colubrina glandulosa Perkins & SI \\
\hline ROSACEAE & Prunus sellowii Koehne & SI \\
\hline RUBIACEAE & $\begin{array}{l}\text { Amaioua guianensis Aubl. } \\
\text { Bathysa nicholsonii K.Schum. } \\
\text { Coutarea hexandra K.Schum. } \\
\text { Guettarda viburnoides Cham. \& Schltdl. } \\
\text { Psychotria carthagenensis Jacq. } \\
\text { Psychotria conjugens Müll.Arg. } \\
\text { Psychotria myriantha Müll.Arg. } \\
\text { Psychotria sessilis (Vell.) Müll. Arg. } \\
\text { Simira sampaioana (Standl.) Steyerm. }\end{array}$ & $\begin{array}{l}\text { SI } \\
\text { SI } \\
\text { SI } \\
\text { SI } \\
\text { PI } \\
\text { ST } \\
\text { ST } \\
\text { SI } \\
\text { SI }\end{array}$ \\
\hline RUTACEAE & $\begin{array}{l}\text { Zanthoxylum rhoifolium Lam. } \\
\text { Hortia arborea Engl. }\end{array}$ & $\begin{array}{l}\text { PI } \\
\text { ST }\end{array}$ \\
\hline SAPINDACEAE & $\begin{array}{l}\text { Allophylus edulis Radlk. ex Warm. } \\
\text { Allophylus sericeus Radlk. } \\
\text { Cupania vernalis Cambess. } \\
\text { Matayba elaeagnoides Radlk. }\end{array}$ & $\begin{array}{l}\text { SI } \\
\text { SI } \\
\text { SI } \\
\text { SI }\end{array}$ \\
\hline SAPOTACEAE & $\begin{array}{l}\text { Chrysophyllum flexuosum Mart. } \\
\text { Chrysophyllum gonocarpum Engl. }\end{array}$ & $\begin{array}{l}\text { ST } \\
\text { ST }\end{array}$ \\
\hline SOLANACEAE & Cestrum amictum Schltdl. & PI \\
\hline SYMPLOCACEAE & Symplocos pubescens Benth. & SI \\
\hline TILIACEAE & Luehea grandiflora Mart. & PI \\
\hline
\end{tabular}

Com base no histórico de proteção, os fragmentos estudados por Meira-Neto e Martins (2000), Silva et al. (2000) e Soares Júnior (2000) e o estudado no presente trabalho apresentaram-se em melhor estado de conservação que aqueles estudados por Almeida \& Souza (1997), Senra (2000) e Meira-Neto et al. (1997). Apesar dos diferentes graus de perturbação, considerando esses trabalhos, na Zona da Mata de Minas Gerais destacaramse como as mais ricas as famílias Annonaceae, Euphorbiaceae, Flacourtiaceae, Lauraceae, Leguminosae, Melastomataceae, Myrtaceae e Rubiaceae, com amplo destaque para Leguminosae. Esses dados reforçam a importância dessas famílias nas Florestas Estacionais, como já tinha chamado a atenção Leitão Filho (1982). 
Quanto aos gêneros mais comumente encontrados nos levantamentos anteriormente analisados para famílias, nos fragmentos mais bem preservados destacaram-se Casearia, Eugenia, Guatteria, Machaerium, Ocotea e Psychotria. Além desses gêneros, excetuando-se Guatteria, nos fragmentos que passaram por interferências mais recentes destacaram-se também Croton, Inga e Nectandra.

Cerca de $70 \%$ desses levantamentos apresentaram 28 espécies em comum (Quadro 2), o que as coloca como características da região, uma vez que foram encontradas em fragmentos em estados de conservação diferentes. Desse total de espécies, apenas Machaerium nyctitans (Vell.) Benth. (Leguminosae Papilionoideae) e Eugenia leptoclada Berg (Myrtaceae) não foram relacionadas no presente trabalho.

Dentre os trabalhos anteriormente analisados, o trecho de floresta do presente estudo foi o que apresentou a maior riqueza em espécies arbóreas, excetuando-se o de Meira-Neto \& Martins (2000), cujo critério de inclusão foi de CAP maior ou igual a $10 \mathrm{~cm}$.

Quadro 2 - Espécies arbóreas mais freqüentemente encontradas em sete levantamentos realizados na Zona da Mata de Minas Gerais. A = Almeida \& Souza (1997), B = Meira-Neto et al. (1997), C = Senra (2000), D = Meira-Neto \& Martins (2000), E = Silva et al. (2000), F = Soares Júnior (2000) e G = presente trabalho

Table 2 - Tree species more frequently found in seven surveys in Zona da Mata (Minas Gerais, Brazil). A = Almeida \& Souza (1997), B = Meira-Neto et al. (1997), C = Senra (2000), D = Meira-Neto \& Martins (2000), $E=$ Silva et al. $(2000), F=S o a r e s$ Júnior (2000) and $G=$ present work

\begin{tabular}{|c|c|c|c|c|c|c|c|}
\hline Espécie & A & $\mathrm{B}$ & $\mathrm{C}$ & $\mathrm{D}$ & E & $\mathrm{F}$ & G \\
\hline Allophylus edulis Radlk. ex Warm. & $\mathrm{x}$ & & $\mathrm{x}$ & $\mathrm{x}$ & $\mathrm{x}$ & & $\mathrm{x}$ \\
\hline Anadenanthera colubrina (Vell.) Brenan & $\mathrm{X}$ & $\mathrm{x}$ & $\mathrm{x}$ & $\mathrm{X}$ & $\mathrm{x}$ & & $\mathrm{x}$ \\
\hline Apuleia leiocarpa J.F.Macbr. & & $\mathrm{X}$ & $\mathrm{x}$ & $\mathrm{x}$ & $\mathrm{x}$ & $\mathrm{x}$ & $\mathrm{x}$ \\
\hline Bathysa nicholsonii K.Schum. & $\mathrm{x}$ & $\mathrm{x}$ & $\mathrm{x}$ & $\mathrm{x}$ & $\mathrm{x}$ & $\mathrm{x}$ & $\mathrm{x}$ \\
\hline Brosimum glaziovii Taub. & & $\mathrm{x}$ & $\mathrm{x}$ & $\mathrm{x}$ & $\mathrm{x}$ & $\mathrm{x}$ & $\mathrm{x}$ \\
\hline Casearia arborea Urb. & & $\mathrm{x}$ & $\mathrm{x}$ & & $\mathrm{x}$ & $\mathrm{x}$ & $\mathrm{x}$ \\
\hline Casearia decandra Jacq. & $\mathrm{x}$ & $\mathrm{x}$ & $\mathrm{x}$ & & $\mathrm{x}$ & $\mathrm{x}$ & $\mathrm{x}$ \\
\hline Casearia ulmifolia Cambess. & & $\mathrm{x}$ & $\mathrm{x}$ & $x$ & $\mathrm{x}$ & $\mathrm{x}$ & $\mathrm{x}$ \\
\hline Copaifera langsdorffii Desf. & & $\mathrm{x}$ & $\mathrm{x}$ & $\mathrm{x}$ & $\mathrm{x}$ & $\mathrm{x}$ & $\mathrm{x}$ \\
\hline Dalbergia nigra Allem. ex Benth. & & $\mathrm{x}$ & $\mathrm{x}$ & $\mathrm{x}$ & $\mathrm{x}$ & $\mathrm{x}$ & $\mathrm{x}$ \\
\hline Erythroxylum pelleterianum A.St.-Hil. & & $\mathrm{x}$ & $\mathrm{x}$ & $\mathrm{x}$ & $\mathrm{x}$ & $\mathrm{x}$ & $\mathrm{x}$ \\
\hline Eugenia leptoclada Berg & & $\mathrm{x}$ & $\mathrm{x}$ & $\mathrm{x}$ & $\mathrm{x}$ & $\mathrm{x}$ & \\
\hline Guapira opposita (Vell.) Reitz & $\mathrm{x}$ & $\mathrm{x}$ & $\mathrm{x}$ & & $\mathrm{x}$ & $\mathrm{x}$ & $\mathrm{x}$ \\
\hline Hortia arborea Engl. & $\mathrm{x}$ & $\mathrm{x}$ & $\mathrm{x}$ & & $\mathrm{x}$ & $\mathrm{x}$ & $\mathrm{x}$ \\
\hline Jacaranda macrantha Cham. & & $\mathrm{x}$ & $\mathrm{x}$ & $\mathrm{x}$ & $\mathrm{x}$ & $\mathrm{x}$ & $\mathrm{x}$ \\
\hline Lacistema pubescens Mart. & & $\mathrm{x}$ & $\mathrm{x}$ & $\mathrm{x}$ & & $\mathrm{x}$ & $\mathrm{x}$ \\
\hline Mabea fistulifera Mart. & $\mathrm{x}$ & $\mathrm{X}$ & $\mathrm{X}$ & $\mathrm{X}$ & $\mathrm{x}$ & $\mathrm{x}$ & $\mathrm{x}$ \\
\hline Machaerium brasiliense Vogel & & $\mathrm{x}$ & $\mathrm{x}$ & $\mathrm{x}$ & $\mathrm{x}$ & & $\mathrm{x}$ \\
\hline Machaerium nyctitans (Vell.) Benth. & $\mathrm{x}$ & $\mathrm{x}$ & $\mathrm{x}$ & & $\mathrm{x}$ & $\mathrm{x}$ & \\
\hline Matayba elaeagnoides Radlk. & $\mathrm{x}$ & $\mathrm{X}$ & $\mathrm{x}$ & $\mathrm{x}$ & $\mathrm{x}$ & $\mathrm{x}$ & $\mathrm{x}$ \\
\hline Ocotea odorifera (Vell.) Rohwer & $\mathrm{x}$ & $\mathrm{x}$ & $\mathrm{x}$ & $\mathrm{x}$ & $\mathrm{x}$ & $\mathrm{x}$ & $\mathrm{x}$ \\
\hline Piptadenia gonoacantha J.F.Macbr. & & $\mathrm{x}$ & $\mathrm{x}$ & $\mathrm{x}$ & $\mathrm{x}$ & $\mathrm{X}$ & $\mathrm{x}$ \\
\hline Pseudopiptadenia contorta (DC.) G.P.Lewis \& M.P.Lima & & $\mathrm{x}$ & & $\mathrm{x}$ & $\mathrm{x}$ & $\mathrm{x}$ & $\mathrm{x}$ \\
\hline Psychotria carthagenensis Jacq. & & $\mathrm{x}$ & $\mathrm{x}$ & $\mathrm{x}$ & & $\mathrm{x}$ & $\mathrm{x}$ \\
\hline Siparuna guianensis Aubl. & $\mathrm{x}$ & $\mathrm{x}$ & $\mathrm{x}$ & $\mathrm{x}$ & $\mathrm{x}$ & $\mathrm{x}$ & $\mathrm{x}$ \\
\hline Sorocea bonplandii (Baill.) Burger, Lanj. \& Boer & $\mathrm{x}$ & & $\mathrm{x}$ & $\mathrm{x}$ & $\mathrm{x}$ & $\mathrm{x}$ & $\mathrm{x}$ \\
\hline Vernonia diffusa Less. & $\mathrm{x}$ & $\mathrm{x}$ & $\mathrm{x}$ & $\mathrm{x}$ & & & $\mathrm{x}$ \\
\hline Zanthoxylum rhoifolium Lam. & $\mathrm{x}$ & $\mathrm{x}$ & & & $\mathrm{x}$ & $\mathrm{x}$ & $\mathrm{x}$ \\
\hline
\end{tabular}


Avaliou-se que cerca de $15 \%$ das espécies encontradas pertencem ao grupo das pioneiras, $53 \%$ ao das secundárias iniciais e $25 \%$ ao das secundárias tardias, devendo ser ressaltado que $6 \%$ das espécies não foram classificadas devido ao desconhecimento de suas características e que Siparuna guianensis foi considerada como indiferente (Quadro 1). Esses dados evidenciam estar o fragmento em estádio intermediário de desenvolvimento. O corte raso praticado na década de 20 e o estádio atual deste fragmento mostram que os dois processos naturais que poderiam promover a recomposição da vegetação, o banco de sementes e a importação de diásporos são eficazes quando a área é mantida sob proteção permanente.

\section{AGRADECIMENTO}

Ao Engenheiro Florestal Renato Ribeiro Mendes, pela participação no primeiro ano de desenvolvimento do presente trabalho; aos especialistas Gilberto Pedralli (Lauraceae), Jorge Yoshio Tamashiro (Leguminosae) e José Rubens Pirani (Meliaceae); e ao Conselho Nacional de Pesquisa (CNPq), pelo auxílio financeiro.

\section{REFERÊNCIAS BIBLIOGRÁFICAS}

ALMEIDA, D. S.; SOUZA, A. L. Florística e estrutura de um fragmento de Floresta Atlântica, no município de Juiz de Fora, Minas Gerais. Revista Árvore, v. 21, n. 2, p. 221230, 1997.

BRUMITT, R. K.; POWELL, C. E. Authors of plant names. Whitstable, Kent, Great Britain: Royal Botanic Gardens - Kew, Whitstable Litho, 1992. 732 p.

CASTRO, P. S. et al. Interceptação da chuva por mata natural secundária na região de Viçosa, MG. Revista Árvore, v. 7, n. 1., p. 76-88, 1973.

CRONQUIST, A. An integrated system of classification of flowering plants. New York: Columbia University, 1981. $1.262 \mathrm{p}$.

GANDOLFI, S. Estudo florístico e fitossociológico de uma floresta residual na área do aeroporto internacional de São Paulo, município de Guarulhos, SP. Campinas: Universidade Estadual de Campinas, 1991. 232 p. Dissertação (Mestrado em Ciências Biológicas) Universidade Estadual de Campinas, 1991.

GOLFARI, L. Zoneamento ecológico do Estado de Minas Gerais para reflorestamento. Belo Horizonte: PRODEPEF/ PNUD/FAO/IBDF, 1975. 65 p. (Série técnica, 3).
LEITÃO FILHO, H. F. Aspectos taxonômicos das florestas do estado de São Paulo. In: CONGRESSO NACIONAL SOBRE ESSÊNCIAS NATIVAS, 1982, Anais... Silvicultura em São Paulo - Instituto Florestal, 1982. v.16 A, pt. 1, p. 197-206.

MARTINS, F. R. Estrutura de uma floresta mesófila. Campinas: Universidade Estadual de Campinas, 1991. 246 p.

MEIRA-NETO, J. A. A.; MARTINS, F. R. Estrutura da Mata da Silvicultura, uma Floresta Estacional Semidecidual Montana no município de Viçosa - MG. Revista Árvore, v. 24, n. 2, p. 151-160, 2000.

MEIRA-NETO, J. A. A. et al. Estrutura de uma Floresta Estacional Semidecidual Submontana em área diretamente afetada pela Usina Hidrelétrica de Pilar, Ponte Nova, Zona da Mata de Minas Gerais. Revista Árvore, v. 21, n. 3, p. 337-344, 1997.

OLIVEIRA-FILHO, A.T. et al. Tree species distribution along soil catenas in a riverside semideciduous forest in southeastern Brazil. Flora, v. 192, p. 47-64, 1997.

OLIVEIRA-FILHO, A. T.; MACHADO, J. N. M. Composição florística de uma floresta semidecídua montana, na serra de São José, Tiradentes, Minas Gerais. Acta Botanica Brasilica, v. 7, p. 71-88, 1993.

OLIVEIRA-FILHO, A.T. et al. Comparison of the woody flora and soils of six areas of montane semideciduous forests in southern Minas Gerais, Brazil. Edinburgh Journal of Botany, v. 51, p. 141-194, 1994.

PAULA, A. Alterações florísticas e fitossociológicas da vegetação arbórea numa floresta estacional semidecidual em Viçosa - MG. Viçosa, MG: Universidade Federal de Viçosa, 1999. 87 p. Dissertação (Mestrado em Botânica) Universidade Federal de Viçosa, 1999.

ROYAL BOTANIC GARDENS - KEW. Index Kewensis on compact disc - manual. Oxford: Oxford University Press, 1997. (CD Room)

SENRA, L. C. Composição florística e estrutura fitossociológica de um fragmento florestal da Fazenda Rancho Fundo, na Zona da Mata - Viçosa, MG. Viçosa, MG: Universidade Federal de Viçosa, 2000. 66 p. Dissertação (Mestrado em Botânica) - Universidade Federal de Viçosa, 2000. 
SILVA, A. F. et al. Composição florística e estrutura horizontal do estrato arbóreo de um trecho da Mata da Biologia da Universidade Federal de Viçosa - Zona da Mata de Minas Gerais. Revista Árvore, v. 24, n. 4, p. 397-405, 2000.

SOARES JÚNIOR, F. J. Composição florística e estrutura de um fragmento de Floresta Estacional Semidecidual na Fazenda Tico-Tico, Viçosa, MG. Viçosa, MG: Universidade Federal de Viçosa, 2000. 68 p. Dissertação (Mestrado em Botânica) - Universidade Federal de Viçosa, 2000.

VALVERDE, O. Estudo regional da Zona da Mata de Minas Gerais. Revista Brasileira de Geografia, v. 20, n. 1, p. 1-82, 1958.
VELOSO, H. P.; RANGEL FILHO, A. L. R.; LIMA J. C. A. Classificação da vegetação brasileira adaptada a um sistema universal. Rio de Janeiro: Instituto Brasileiro de Geografia e Estatística, 1991. 124 p.

VIANELLO, R. L.; ALVES, A. R. Meteorologia básica e aplicações. Viçosa, MG: Universidade Federa de Viçosa, 1991. $448 \mathrm{p}$.

WILCOVE, D. S. Habitat fragmentation in temperate zones. In: SOULÉ, M. E. (Ed.) Conservation biology. Massachusetts: Sinauer Press, 1986. p. 237-256. 